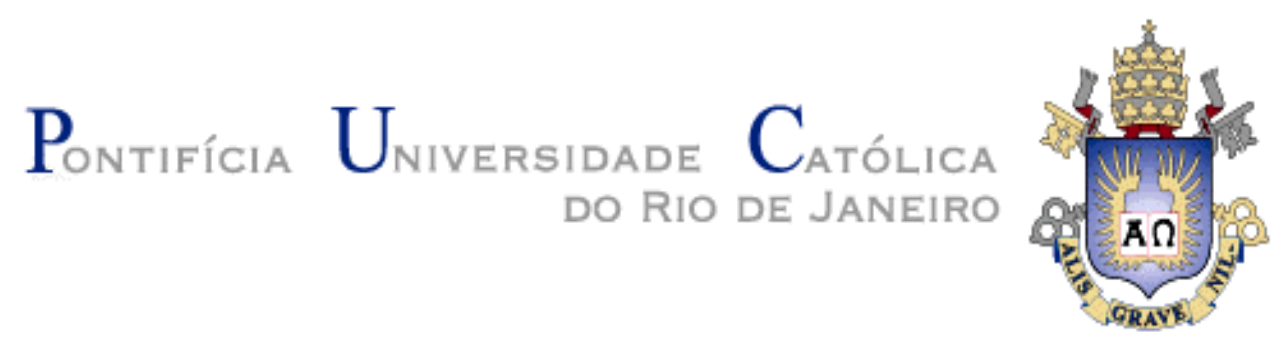

Gustavo Silvano Batista

A relevância da arquitetura no pensamento de Gadamer

Tese de Doutorado

Tese apresentada ao Programa de Pós-graduação em Filosofia do Departamento de Filosofia da PUC-Rio como parte dos requisitos parciais para obtenção do título de Doutor em Filosofia.

Orientador: Prof. Paulo Cesar Duque-Estrada Co-orientadora: Prof. ․ Lígia Teresa Saramago Pádua

Rio de Janeiro

Dezembro de 2013 
Pontifícia U Universidade C $_{\text {atólica }}$

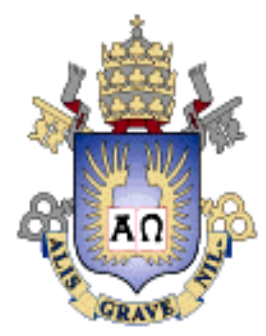

Gustavo Silvano Batista

\section{A Relevância da Arquitetura no Pensamento de Gadamer}

Tese apresentada como requisito parcial para obtenção do grau de Doutor pelo Programa de Pósgraduação em Filosofia do Departamento de Filosofia do Centro de Teologia e de Ciências Humanas da PUC-Rio. Aprovada pela Comissão Examinadora abaixo assinada.

Prof. Paulo Cesar Duque-Estrada

Orientador

Departamento de Filosofia - PUC-Rio

Profㄹ. Lígia Teresa Saramago Pádua

Co-orientadora

Departamento de Filosofia - PUC-Rio

Prof. Fernando Antônio Soares Fragozo

Escola de Comunicação - UFRJ

Prof. Alessandro Rodrigues Pimenta Departamento de Filosofia - UFPI

Profa. Dirce Eleonora Nigro Solis Departamento de Filosofia - UERJ

Prof. Edgar de Britto Lyra Netto Departamento de Filosofia - PUC-Rio

Profa. Denise B. Portinari Coordenadora Setorial do Centro de Teologia e de Ciências Humanas - PUC-Rio

Rio de Janeiro, 02 de dezembro de 2013 
Todos os direitos reservados. É proibida a reprodução total ou parcial do trabalho sem autorização da universidade, do autor e do orientador.

\section{Gustavo Silvano Batista}

Graduou-se em Filosofia (2004) na PUC-Rio, onde também fez o Mestrado em Filosofia (2007). É Professor da Universidade Federal do Piauí - UFPI. Foi visiting scholar no Boston College - EUA (2010-2011). Cursou o Doutorado em Filosofia na PUC-Rio (2010 2013) e Doutorado Sanduíche no Departamento de Artes e Estudos Culturais da Københavns Universitet (2012-2013) - Dinamarca, obtendo o título de doutor com a presente tese.

Ficha Catalográfica

Batista, Gustavo Silvano

A relevância da arquitetura no pensamento de Gadamer / Gustavo Silvano Batista ; orientador: Paulo Cesar Duque-Estrada ; co-orientadora: Lígia Teresa Saramago Pádua. - 2013.

123 f. : il. (color.) ; $30 \mathrm{~cm}$

Tese (doutorado)-Pontifícia Universidade Católica do Rio de Janeiro, Departamento de Filosofia, 2013.

Inclui bibliografia

1. Filosofia - Teses. 2. Gadamer. 3. Arte. 4. Ontologia. 5. Arquitetura. 6. Representação. 7. Compreensão. I. Duque-Estrada, Paulo Cesar. II. Pádua, Ligia Teresa Saramago. III. Pontifícia Universidade Católica do Rio de Janeiro. Departamento de Filosofia. IV. Título. 
Este trabalho é dedicado a Paulo Cesar Duque-Estrada, com amizade. 


\section{Agradecimentos}

Ao meu orientador, Professor Paulo Cesar Duque-Estrada, a quem dedico esta tese, pelo incentivo e apoio incondicionais. Agradeço também sua amizade e por acompanhar por tantos anos meu desenvolvimento intelectual com o mesmo entusiasmo.

À minha co-orientadora, Professora Lígia Saramago, por sua amizade, generosidade intelectual e interesse em contribuir com minha formação acadêmica.

Aos professores do Departamento de Filosofia da PUC-Rio, de modo especial aos professores Deborah Danowski, Luiz Camillo Osório e Vera Cristina Bueno, pelo apoio e gentileza.

Ao Professor Pe. Paul Schweitzer SJ, do Departamento de Matemática da PUC-Rio, pelo apoio por ocasião do período de pesquisa no Boston College.

Ao Prof. Jeffrey Bloechl, pela acolhida, amizade e orientação nos meses que estive no Boston College. Agradeço também aos professores James Bernauer SJ, Richard Kearney, John Sallis e à Professora Catherine Cornille, do Departamento de Teologia, pela atenção, diálogo e convívio inesquecíveis durante o duro período de inverno em Chestnut Hill.

Ao Professor Richard J. Bernstein, pela calorosa acolhida na New School for Social Research e pelo longo e entusiasmado diálogo, que muito contribuiu para a escrita desta tese.

Ao Professor Abdulrazack Karriem, pela acolhida em Ithaca, NY e também pela amizade e apoio incondicionais. Agradeço por me inserir no excelente ambiente acadêmico da Cornell University, pelo acesso à Fine Arts Library e à John M. Olin Library.

Ao Professor Karl Erik Schøllhammer, pela amizade e oportunidade de realização dos meus estudos em Copenhague. Serei para sempre grato por me proporcionar um período de grande amadurecimento intelectual e humano.

Ao Professor Anders Michelsen, pela acolhida e orientação nos meses que estive na Københavns Universitet. Agradeço também a acolhida dos Professores Mette Sandbye e Frederik Tygstrup e ao apoio gentil e decisivo de Thomas Klenow, Kristine Søndergaard e Claudius Pratt durante os meses gelados, escuros e inesquecíveis no Vesterbro. 
Ao Professor Henrik Øxvig, da Escola de Arquitetura da The Royal Danish Academy of Fine Arts, pelo convite para um workshop com o Professor Gottfried Boehm em Basiléia.

Ao Professor Rico Sneller, pela acolhida em Leiden e pelo diálogo sobre Gadamer durante nossa caminhada na Universiteit Leiden.

Aos queridos colegas do doutorado: Maria Priscilla Coelho e Thiago Faria, pela amizade e por compartilharmos interesses filosóficos diversos.

Aos amigos que encontrei no Piauí: Alessandro Pimenta, Ana Márcia Almeida, Ana Paula Cantelli, André Gonçalves, Cristiane Lopes, Erik Rodarte, Francisco Tadeu Fontenele, Gilvana Pessoa, Helder Buenos Aires de Carvalho, João Gabriel Cavalcante, Júlia Sampaio, Leandro Sardeiro, Rodrigo Gerolineto e Suze Sales.

Aos amigos de sempre, sempre resistentes à distância: Daniel Siquara, Diogo Corrêa, Evandro José, Fabiano Ferreira, Fátima Borges, Felipe Konzen, Fernando Cruz, Frederico Secco, Karina Lelles, Linovaldo Lemos, Lúcia Lanna, Luiz Alberto Couceiro, Maria Auxiliadora Teixeira, Maria Luiza Oswald, Margareth Urbano, Pedro Belchior, Rogério Acca, Renato Marques, Rejane Valvano, Roberto Azevedo, Roberto Wu, Wellington Campos e Zuleica Strogulski.

A dois amigos que não estão mais entre nós, mas sempre presentes em minhas lembranças: Santuza Cambraia Naves e Rubens Silvano.

A minha família, de modo especial aos meus pais, Antonio Reis e Maria Gilda Silvano, e a minha irmã, Maria Madalena Silvano, pela presença sempre atenta e cordial.

A Edna Sampaio e a Diná Santos, pela gentileza de sempre.

Aos professores da banca examinadora. Agradeço a disponibilidade na leitura e avaliação desta tese e também pelos preciosos comentários e sugestões.

A CAPES, pela concessão da bolsa sanduíche na Dinamarca e à Vice-Reitoria Acadêmica da PUC-Rio, pela bolsa de isenção, sem a qual este trabalho não poderia ter sido realizado. 


\section{Resumo}

Batista, Gustavo Silvano; Pádua, Lígia Saramago. Duque Estrada, Paulo Cesar; A relevância da arquitetura no pensamento de Gadamer. Rio de Janeiro, 2013. 123p, Tese de Doutorado - Departamento de Filosofia, Pontifícia Universidade Católica do Rio de Janeiro.

Na primeira parte de Verdade e Método, Gadamer dedica-se a examinar a arte como uma das esferas privilegiadas na qual a verdade acontece de modo alternativo ao modelo científico estabelecido. Ao descrever os elementos constitutivos da experiência da verdade na obra de arte, Gadamer apresenta a arquitetura como um modelo exemplar, pois nela a referida experiência da verdade acontece de um modo bastante peculiar. Ou seja, a experiência hermenêutica da arquitetura serve de paradigma para a explicitação de um devido questionamento do acontecimento da compreensão, já que não perde de vista a esfera ético-prática na qual se encontra inserida. Deste modo, esta tese tem como objetivo analisar a abordagem gadameriana da arquitetura, no sentido de afirmar seu caráter filosoficamente relevante no cerne da estrutura do projeto de Gadamer. Na relação com as obras arquitetônicas, podemos notar traços hermenêuticos fundamentais que sustentam uma experiência de pensamento que não se submete ao modelo representativo próprio da ciência, mas o contesta.

\section{Palavras-chave}

Gadamer; arte; ontologia; arquitetura; representação; compreensão. 


\section{Abstract}

Batista, Gustavo Silvano; Pádua, Lígia Saramago (Co-advisor); Duque Estrada, Paulo Cesar (Advisor). The relevance of architecture on Gadamer's thought Rio de Janeiro, 2013. 123 p. Phd. Thesis Departamento de Filosofia, Pontifícia Universidade Católica do Rio de Janeiro.

In the first part of Truth and Method, Gadamer dedicates to discuss the art as one of privileged spheres where the truth happens alternatively the scientific model established. To describe elements of the experience of truth in the artwork, Gadamer presents the architecture as a model, because in it the same experience of truth peculiarly happens. In other words, the hermeneutic experience of architecture serves as a paradigm for the explanation of a righteous questioning of the event of understanding, insered in the ethical-practical sphere of life. Thus, this thesis aims to analyze the Gadamer's approach of architecture, affirming its character philosophically relevant at the heart of the structure of Gadamer's thought. So, in relation with architectural works, we can see hermeneutic traces that support thought experiences that no submit to the representative model of science but contests it.

\section{Keywords}

Gadamer; art; ontology; architecture; representation; understanding. 


\section{Sumário}

1. Introdução 14

2 - Compreensão e crítica da representação: Gadamer, sua herança filosófica e a experiência hermenêutica da obra de arte 17

2.1 - Gadamer e a arte: em diálogo com Hartmann e Heidegger 20

2.2 - Hermenêutica filosófica e a superação da estética 34

2.3 - Compreensão e crítica da representação 40

3 - O caráter relevante da arquitetura em Verdade e Método e nos escritos posteriores $\quad 45$

3.1 - A arquitetura em Verdade e Método 50

3.2 - A arquitetura nos escritos posteriores 63

3.2.1 - A Imagem Emudecida 63

3.2.2 - A atualidade do Belo: a arte como jogo, símbolo e festa 66

$\begin{array}{ll}3.2 .2 .1 \text { - Jogo } & 70\end{array}$

$\begin{array}{ll}\text { 3.2.2.2 - Símbolo } & 74\end{array}$

$\begin{array}{ll}\text { 3.2.2.3 - Festa } & 77\end{array}$ 
$\begin{array}{ll}\text { 3.2.4 - Palavra e Imagem } & 87\end{array}$

4. Hermenêutica filosófica e teoria da arquitetura: Dalibor Vesely e a herança de Gadamer

5. Conclusão

6. Bibliografia 


\section{Lista de figuras}

Figura 1 - Casa da Música, Porto - Portugal 48

Figura 2 - Casa da Música, Porto - Portugal 48

Figura 3 - Casa da Música, Porto - Portugal 48

Figura 4 - Casal Arnolfini - National Gallery, Londres - UK 57

Figura 5 - Catedral de St. Gallen, Gallen - Suíça 83

Figura 6 - Catedral de St. Gallen, Gallen - Suíça 83

Figura 7 - Pintura Rupestre da Serra da Capivara - Piauí 90

Figura 8 - Palácio Gustavo Capanema, Rio de Janeiro 93

Figura 9 - Palácio Gustavo Capanema, Rio de Janeiro 93

Figura 10 - Palácio Gustavo Capanema, Rio de Janeiro 94

Figura 11 - Palácio Gustavo Capanema, Rio de Janeiro 94 


\section{Abreviaturas das obras de Gadamer utilizadas nesta tese}

$\mathrm{AB}$ - A Atualidade do Belo: a arte como jogo, símbolo e festa

IE - Imagem Emudecida

INT - Introdução à Origem da Obra de Arte

LCQ - Sobre a leitura de construções e quadros

PA - Philosophical Apprenticeships

$\mathrm{PI}$ - Palavra e Imagem

PCH - O Problema da Consciência Histórica

VM - Verdade e Método 
Somente o que pode ser representado pode ser construído. Verbete 'Representar', The Metapolis Dictionary of Advanced Architecture.

As obras arquitetônicas não permanecem irreversíveis, à margem do fluxo histórico da vida, mas este arrasta-as consigo. Hans-Georg Gadamer, Verdade e Método. 